\title{
Haptic Inspection of Planetary Soils with Legged Robots
}

\section{Journal Article}

Author(s):

Kolvenbach, Hendrik; Bärtschi, Christian; Wellhausen, Lorenz (D); Grandia, Ruben; Hutter, Marco (D)

Publication date:

2019-04

Permanent link:

https://doi.org/10.3929/ethz-b-000322720

Rights / license:

In Copyright - Non-Commercial Use Permitted

Originally published in:

IEEE Robotics and Automation Letters 4(2), https://doi.org/10.1109/LRA.2019.2896732

Funding acknowledgement:

780883 - subTerranean Haptic INvestiGator (EC) 


\title{
Haptic Inspection of Planetary Soils with Legged Robots
}

\author{
Hendrik Kolvenbach, Christian Bärtschi, Lorenz Wellhausen, Ruben Grandia and Marco Hutter
}

\begin{abstract}
Planetary exploration robots encounter challenging terrain during operation. Vision-based approaches have failed to reliably predict soil characteristics in the past, making it necessary to probe the terrain tactilely. We present a robust, haptic inspection approach for a variety of fine, granular media, which are representative of Martian soil. In our approach, the robot uses one limb to perform an impact trajectory, while supporting the main body with the remaining three legs. The resulting vibration, which is recorded by sensors placed in the foot, is decomposed using the discrete wavelet transform and assigned a soil class by a Support Vector Machine. We tested two foot designs and validated the robustness of this approach through the extensive use of an open-source dataset, which we recorded on a specially designed single-foot testbed. A remarkable overall classification accuracy of more than $98 \%$ could be achieved despite various introduced disturbances. The contributions of the different sensors to the classification performance are evaluated. Finally, we test the generalization performance on unknown soils and show that the interaction behavior can be anticipated.
\end{abstract}

Index Terms-Force and Tactile Sensing, Space Robotics and Automation, Legged Robots, AI-Based Methods

\section{INTRODUCTION}

$\mathbf{T}$ RAVERSING fine-grained, granular terrain is a challenging task for mobile robots due to the difficulty of modeling and anticipating terramechanic effects for control. Especially during robotic exploration of sandy, remote, and inaccessible environments, such as the Moon and Mars, estimating the terrain properties before traversal is crucial in order to avoid mission hazards or to find an energy-efficient path.

Purely visual analysis of the soil is risky and has caused significant delays during missions with the Mars Exploration Rovers (MER) since the subsurface composition and physical properties of the soil remain largely unknown [1] [2] [3]. For example, NASA's Spirit rover got immobilized on Mars after breaking through a solid-looking, crusty surface layer (duricrust) into the soft sand hidden underneath [3]. Notably, a recent study using thermal data has found a correlation between visual thermal data and slip behavior of the Curiosity rover, yet the robustness of the approach remains to be evaluated [4].

Manuscript received September 07, 2018; Revised December, 04, 2018; accepted January 10, 2019.

This paper was recommended for publication by Editor Jonathan Roberts upon evaluation of the Associate Editor and Reviewers' comments.

This work was supported by the European Space Agency (ESA) and Airbus DS in the framework of the Network Partnering Initiative 481-2016 and the European Unions Horizon 2020 research and innovation programme under grant agreement No 780883 .

All the authors are with the Robotic Systems Lab (RSL) at ETH Zurich, Switzerland hendrik.kolvenbachemavt.ethz.ch

Digital Object Identifier (DOI): see top of this page.

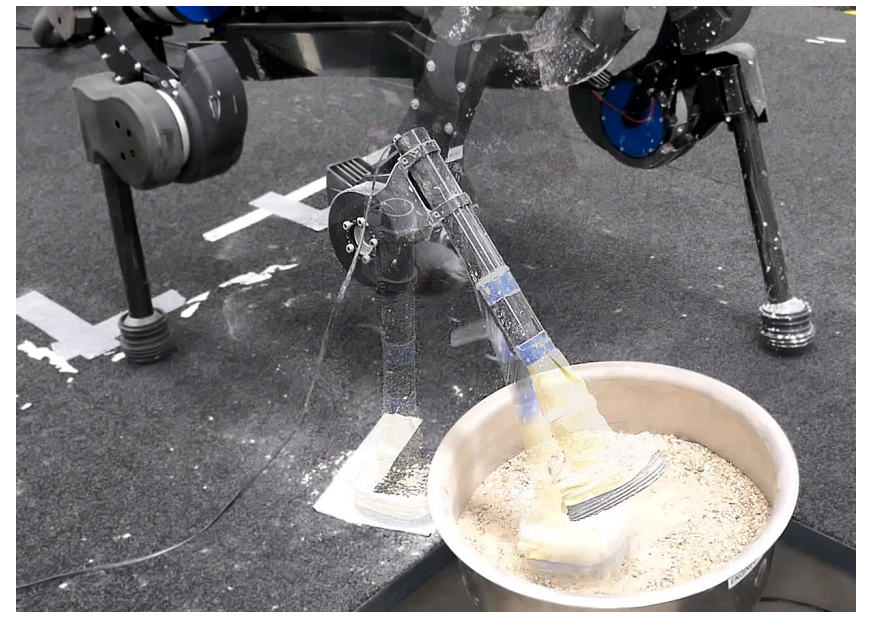

Fig. 1: ANYmal is inspecting planetary soil in front of the system. The robot executes an impact motion with a specially designed, sensor-equipped foot while keeping the remaining feet in a safe stance.

Traditionally, sinkage and shear tests are used to extract terramechanic parameters [5]. These parameters are used in robot-soil interaction models (i.e. for wheels [6] or legs [7]) to predict the robot's behavior. However, uncertainty exists in the terrain parameter estimation and the interaction models, leading to gaps between observed and estimated performances.

A promising practical approach is to use machine learning techniques to classify terrain types based directly on experimental observations and to adapt the robot's motion accordingly. Supervised classification of Martian soils for wheeled systems has been investigated in the past using vibration response [8] combined with visual data from the robot for supervised [9] and unsupervised training [10].

Supervised classification of Martian soils has also been performed with a single leg-wheel hybrid [11]. The wheel consists of five equally spaced 'feet' which are rigidly connected to a rimless, spoked wheel. An Inertial Measurement Unit (IMU) on the stator frame was used to predict slip, while a camera observing the terrain measured the sinkage. We consider this work to be closer to wheeled locomotion than legged locomotion because of the mechanical design of the wheel with rigid feet.

The supervised classification of terrestrial surfaces has been investigated for legged systems with the use of machine learning techniques such as Support Vector Machines [12] [13] [14] [15], AdaBoost [16], Discriminant Analysis [17], Pitman Yor Process Mixture of Gaussians [18], Neural Networks [19] and Random Forests [20]. A good review of terrain classification methods for legged robots is given by Kertsz [20]. 
All authors investigated a broad variety of terrestrial materials such as rubber tiles, carpets, gravel, concrete etc. but only some included a sand or dirt class which is not further specified [12] [17] [18] [21]. Therefore, a detailed analysis of the applicability of classification techniques for legged systems on a variety of fine-grained, granular media in which terramechanic effects dominate is missing.

So far, studies focused on (offline) classification based on a pre-recorded training set of a walking robot and only a few included online classification with locomotion parameter adaptation [12] [14]. In all cases, the robot was already in full interaction with the terrain when classification took place. Additionally, the authors of [15] found that the performance of a classifier is largely depended on gait and velocity, increasing the chance of errors due to a large number of varying parameters when performing classification while walking.

If the safety of the robotic system is a concern, such as during extraterrestrial exploration, one would want to know the safety of a foothold a priori. Similar to humans using their limbs to inspect the temperature of a lake before swimming or the slippage or rigidity of an icy patch, we want to use the limbs of a legged robot to safely inspect the terrain. To our knowledge, only our group has investigated a dedicated, single scratching motion on a single-leg testbed to classify hard surfaces based on qualitative measurements, as seen in the work of Höpflinger et al [13]. Thus, using the limbs of an entirely controlled robot to perform a dedicated, haptic inspection of the environment is not demonstrated so far. In this context, it remains an open question how such a foot for soil inspection should be designed.

In this paper, we present a robust classification approach for dry, granular media which is representative of Martian soils by using the specifically designed, sensor-equipped feet of a quadruped robot. We evaluate a point foot design and a passiveadaptive planar foot design and assess the individual sensor contributions to the classification performance. We introduce a foot-soil interaction testbed with a tiltable soil container, with which we have collected and open-sourced a large dataset on different soils in order to test the robustness of our approach quantitatively. We also demonstrate a haptic inspection motion with a quadruped robot which results in immediate and accurate knowledge about anticipated terrain behavior without putting the system at risk. This work is part of a continuing effort to raise the technology readiness level of dynamically walking robots for space exploration [22] [23].

\section{A. Paper outline}

We describe the design of a sensorized point and adaptive foot in Sec. II and introduce the testbed, the inspection maneuver and the soils investigated in this study in Sec. III. Subsequently, we present the test procedure and the classification method in Sec. IV, while we present the results in Sec. V. Finally, we conclude the work in Sec. VI.

\section{POINT AND PLANAR FOOT DESIGN}

\section{A. Point foot design}

Simplistic point feet are widely used for quadruped robots. Our robot, ANYmal [24] uses point feet consisting of a nitrile

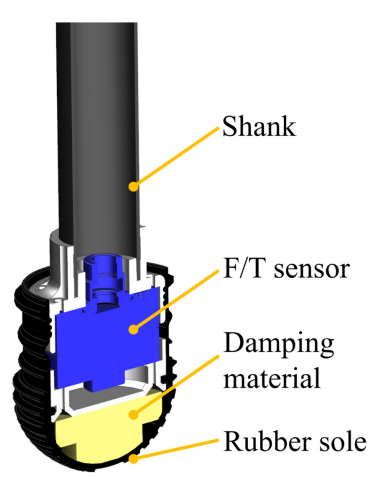

(a) Point foot

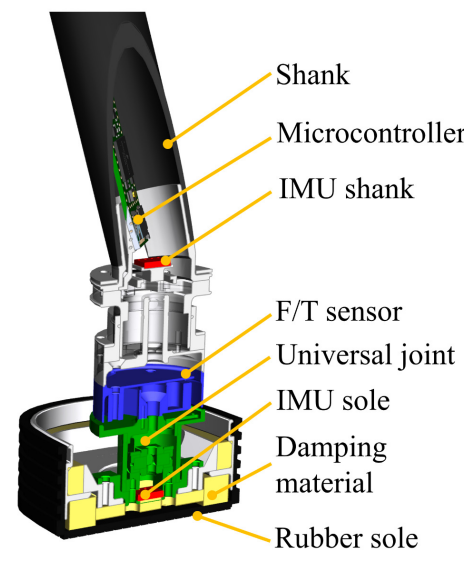

(b) Planar foot
Fig. 2: Cross-section view of the sensor-equipped point and planar feet utilized in this study.

rubber (NBR) sole of elliptical shape, supported by $15 \mathrm{~mm}$ of memory foam (Poron XRD) to reduce peak loads during impact (Figure 2a). The diameter of a foot typically creates a surface area of $8 \mathrm{~cm}^{2}$ on hard surfaces but can reach up to $28 \mathrm{~cm}^{2}$ if fully submerged in compressible terrain. A foot weighs $325 \mathrm{~g}$ (including shank). We integrated an in-house developed 6-Axis Force/Torque (F/T) sensor in the design for the purpose of this work. The sensor allows for sampling at $400 \mathrm{~Hz}$. While this foot design performs well on most hard terrains, we observed shortcomings on loose and compressible soils due to high sinkage [25].

\section{B. Planar foot design}

We developed a passive adaptive planar foot (Figure $2 b$ ) with a weight of $380 \mathrm{~g}$ (including shank) to increase the robot's mobility on compressible soils [26]. The design features a passive ankle joint which can adapt to the terrain up to $\pm 45^{\circ}$ around pitch axis and $\pm 30^{\circ}$ around the roll axis. We manufactured the foot sole out of a natural/styrene-butadiene rubber (NR/SBR) pad with a surface area of $50 \mathrm{~cm}^{2}$, which is supported by $12 \mathrm{~mm}$ of memory foam (Poron XRD). The foot incorporates two InvenSense ICM-20608-G IMUs in order to measure the relative orientation of the sole. We placed the aforementioned 6-Axis F/T sensor in the shank. The IMU data is sampled at $4 \mathrm{kHz}$ and averaged to $400 \mathrm{~Hz}$. The F/T data is sampled slightly slower at $330 \mathrm{~Hz}$ compared to the point foot due to computational limitations. All signals are collected by an integrated microcontroller and sent via USB at $400 \mathrm{~Hz}$.

\section{EXPERIMENTAL SETUP}

\section{A. Foot-soil interaction testbed}

We manufactured a small-scale foot-soil interaction testbed to collect reproducible datasets in a controlled environment (Figure 3). The testbed is especially valuable since planetary soil simulants are a scarce resource and robot-size testbeds are costly to produce and maintain. The testbed consists of an impact test mechanism and a tilt mechanism which holds the soil containers. 


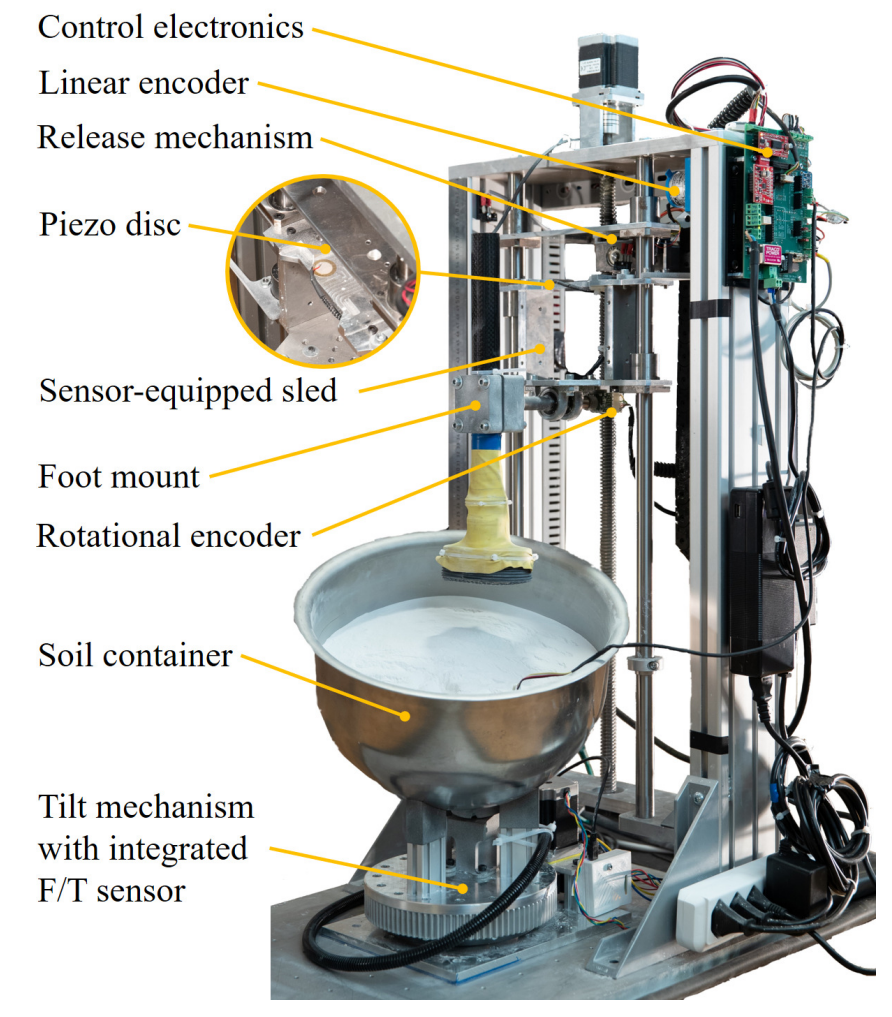

Fig. 3: The foot-soil interaction testbed consists of an impact test mechanism and a tilt mechanism for the exchangeable soil container. The sensor-equipped feet are mounted on the sled. The testbed sensor data and the feet sensor data are collected by a nearby operator PC (not shown).

The impact test mechanism allows for repetitive drop-tests from heights up to $310 \mathrm{~mm}$ (soil surface to the axis of the foot mount) and $560 \mathrm{~mm}$ (base plate to the axis of the foot mount). During testing, a carriage picks up the sled with the attached foot and releases it once it reaches a user-defined height.

The tilt mechanism enables the rotation of the exchangeable soil container between $0^{\circ}-25^{\circ}$ in roll and pitch, allowing us to simulate soil inclinations. The soil containers are of a halfspherical shape to minimize border effects during interaction with the foot. We manufactured five identical soil containers with a common interface which allows us to replace the soil during the dataset acquisition with minimal handling efforts and without the risk of cross-contamination. The tilt mechanism is additionally equipped with an ATI Mini58 6-axis F/T sensor. The impact test mechanism features environmental sensors such as temperature/humidity (Adafruit BME280) and moisture (Sparkfun SEN-13322). The sled is equipped with an analog accelerometer (ADXL 335), a piezo disk, and an absolute linear position (Sick BCG05-C1QM0199) and rotational encoder (AMS AS5048A).

The actuators and sensors, besides the F/T sensor, are connected to a microcontroller on the testbed for real-time data acquisition purposes. The sensors are used for ground truth measurements and for guaranteeing a consistent test environment. The overall system is designed for high stiffness and placed on a shock-absorbing mat so as to avoid oscillations which could disturb the classification.

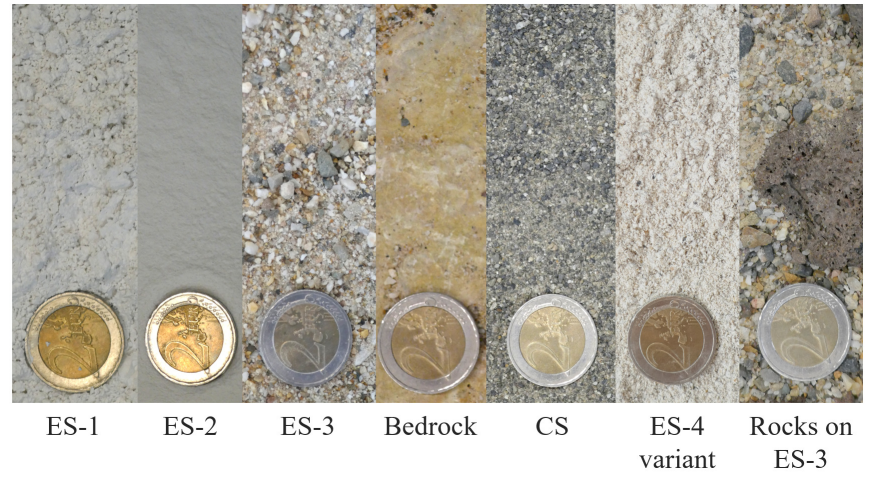

Fig. 4: The soils we investigated in our experiments. The 'bedrock covered in ES-2' is not visually distinguishable from ES-2 and is thus not depicted.

\section{B. Robotic inspection motion}

We recreated the impact motion to inspect unknown terrain with the quadruped robot ANYmal. In our experiment, we placed the robot on the laboratory carpet floor in front of the soil container.

The three feet of the robot guarantee a stable stance, while one limb with the sensor-equipped foot reaches ahead to perform the inspection. By doing so, the robot can safely examine the terrain without the risk of falling. The forcecontrolled motion was created using the "Free Gait" framework [27].

\section{Soils investigated in this study}

The soils used in this study are representative of Martian regolith, which consists of dust, sand, and rocks. The so-called Engineering Soils (ES) were created by industry under the supervision of the European Space Agency based on the analysis of the mechanical properties of regolith encountered during previous Mars missions [5]. The average soil characteristics can be found in Table I. The grains are distributed evenly within the soil. In the following, we give a brief qualitative assessment of the soils and their potential occurrences on Mars.

- ES-1: Very fine-grained, porous and highly compressible dust, which often occurs in the form of patches or as a thin layer on top of other materials.

- ES-2: Silt to very fine sand which appears to be very common on Mars and is found in particular in the troughs of ripples and dunes. This soil was in the past found to be most challenging to traverse with rovers.

- ES-3: A gravelly, medium-coarse sand. The soil is considered representative for scree, coarse aeolian sands, and polymodal surficial lag. Coarse scree and aeolian accumulations occur in terrain with escarpments. Surficial lag occurs where finer grains have been removed by the wind, forming a relatively thin layer which protects finer grains in the subsurface from further erosion [5].

- Bedrock: A solid, rough limestone representative for exposed bedrock on the surface of Mars. In our experiment, a large limestone with a thickness of $40 \mathrm{~mm}$ is rigidly embedded in ES-3 with an exposed surface. 
TABLE I: Physical properties of the soils.

\begin{tabular}{|c|c|c|c|c|}
\hline \multirow{2}{*}{$\begin{array}{c}\text { Soil } \\
\text { properties }\end{array}$} & \multicolumn{4}{|c|}{ Soil type } \\
\cline { 2 - 5 } & ES-1 & ES-2 & ES-3 & CS \\
\hline Appearance & fine dust & fine sand & coarse sand & coarse sand \\
Modal grain size $[\mu \mathrm{m}]$ & $\sim 10$ & - & $\sim 400-600$ & $\sim 1000$ \\
Min grain size $[\mu \mathrm{m}]$ & $<\sim 10$ & $>\sim 30$ & $>\sim 30$ & $>\sim 50$ \\
Max grain size $[\mu \mathrm{m}]$ & $\sim 32$ & $\sim 125$ & $\sim 20.000$ & $\sim 2000$ \\
Int. friction angle $\left[{ }^{\circ}\right]$ & $34 \pm 4$ & $37 \pm 5$ & $35 \pm 5$ & - \\
Cohesion $[\mathrm{kPa}]$ & $1 \pm 0.5$ & $0.75 \pm 0.75$ & $0.15 \pm 0.15$ & - \\
Dry bulk density $\left[\mathrm{g} / \mathrm{cm}^{3}{ }^{3}\right.$ & $1.2 \pm 0.2$ & $1.5 \pm 0.1$ & $1.6 \pm 0.1$ & - \\
\hline
\end{tabular}

In reality on Mars, a mixture of soils can be encountered, and the subsurface composition may vary from the surface layer. Hence, we introduce additional soils to test the generalization capabilities of our approach. We refer to these soils as unknown.

- Coarse sand (CS): A coarse, heterogeneous off-theshelf sand which is not representative of Martian soil. Qualitatively, the soil interaction with the two feet is similar to ES-3.

- Bedrock covered in ES-2: The aforementioned limestone is rigidly embedded in ES-2 and covered by a $5-10 \mathrm{~mm}$ thick surface layer of fine sand, which simulates underlying bedrock, for example in the vicinity of Martian ripples.

- ES-4 variant: ES-4 is a 0.3 mass ratio mixture of ES-1 and ES-3. According to the specifications, the admixed larger grains of gravel within ES-3 should be removed. However, this was not possible in our case so we could only recreate a variation of ES-4. The material behavior is highly dependent on the packing density and varies between very loose, ES-1 type behavior to highly rigid ES-3/bedrock type behavior, which is visually indistinguishable.

- Rocks on ES-3: Volcanic boulders are a common sight on Mars and can disturb classification performance. We placed volcanic rocks of arbitrary shape and with an edge length of a few centimeters in ES-3. The rocks are scattered, not touching, and semi-recessed in the soil. The rocks were repositioned frequently to achieve different contact points.

\section{METHOD}

\section{A. Dataset collection}

We acquired a total of three datasets. Two large datasets were recorded on the single leg testbed for each foot type to systematically evaluate their design and the robustness of the classification against disturbances. A smaller dataset was recorded with the planar foot on the robot ANYmal to show the validity of the inspection motion on a real system.

It is essential to prevent over-fitting the model to extraneous signals, so we took special care concerning the testing procedure. During testing, we kept the filling height of the containers equal and placed small masses deep in the soil to achieve equal weight. We raked the soil with a comb-like tool after each impact. The raking is necessary since the internal friction angle and the cohesion of the soil depend on the

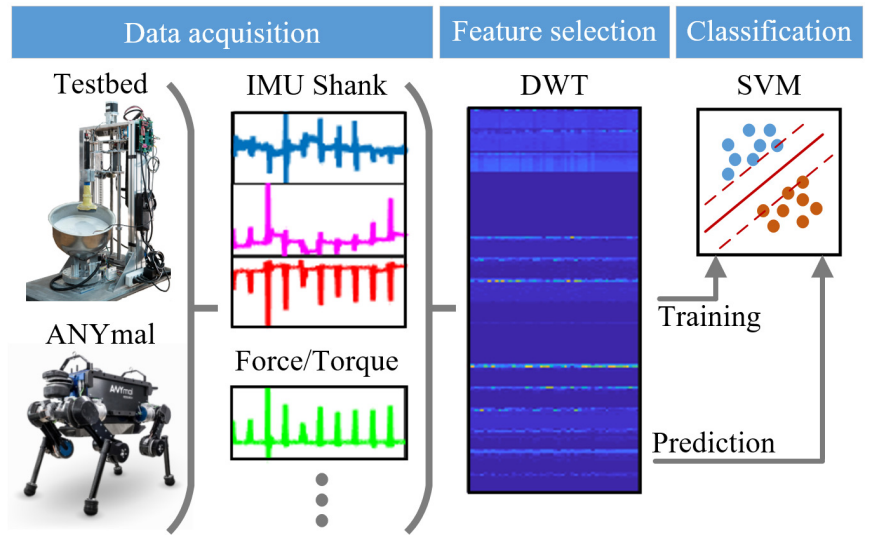

Fig. 5: Schematic overview of the approach we followed in our work.

packing density, which would otherwise increase after each impact. The recreation of similar packing density could only be done qualitatively, but we consider this variety as a challenge for the classification algorithm.

On the testbed, we randomly varied the drop height between $40 \mathrm{~mm}$ to $60 \mathrm{~mm}$ and adjusted the mass of the lift to $1.44 \mathrm{~kg}$ in order to replicate impact velocities and forces which can be achieved by the robot. The roll and pitch axis of the soil container was varied between $0^{\circ}-25^{\circ}$ to simulate realistic conditions. The feet could freely rotate around the lateral axis of the sled, which resulted in slippage at steep inclinations. We randomly exchanged the already-prepared soil containers after a few tests to be invariant to potential sensor drift. During the exchange, the foot sensors are powered, and we carefully cleaned the foot with a brush before continuing the experiment.

A set of 175 impact tests was collected with each foot for each known soil: ES-1, ES-2, ES-3 and the bedrock. Smaller sets of unknown soils were recorded with the same variations: CS, ES-4 (loose), ES-4 (compacted), the embedded bedrock in ES-2 and rocks placed on ES-3. This results in a total amount of 2600 impacts recorded with the testbed.

With the robot, we repeatedly executed the previously mentioned impact trajectory. We performed 50 impacts with the planar foot on non-inclined known soil. A small set of 20 impacts was performed on unknown soil, the CS and the embedded bedrock in ES-2. This results in a total amount of 240 impacts recorded with the robot.

The minimal post-processing of the acquired foot sensor data involved the identification of the impact peak and the extraction of the raw impact oscillation. This results in a signal length of 200 sample points or $0.5 \mathrm{~s}$ per impact. The complete dataset, named PALPATE (Planetary Soil Impact Dataset), is openly available for further investigations ${ }^{\dagger}$.

\section{B. Feature selection}

The acquired data consists of six signals for the point foot (Force/Torque) and 18 signals for the planar foot (Force/Torque, IMU shank (linear acceleration/angular velocity), IMU sole (linear acceleration/angular velocity).

\footnotetext{
${ }^{\dagger}$ http://www.rsl.ethz.ch/publications-sources/datasets
} 
TABLE II: Accuracy of the linear SVM classification related to selected sensor signals for the point* and planar** foot.

\begin{tabular}{|c|c|c|c|c|c|c|}
\hline \multirow{2}{*}{$\begin{array}{c}\text { Sensor } \\
\text { signal }\end{array}$} & No. total & \multicolumn{5}{|c|}{ Classification accuracy } \\
\cline { 3 - 7 } & Features & ES-1 & ES-2 & ES-3 & Bedrock & Overall \\
\hline Force* $^{*}$ & 309 & $100 \%$ & $91 \%$ & $100 \%$ & $100 \%$ & $\mathbf{9 7 . 9 \%}$ \\
Torque* $^{*}$ F/T $^{*}$ & 309 & $97 \%$ & $91 \%$ & $94 \%$ & $100 \%$ & $\mathbf{9 5 . 7 \%}$ \\
\hline FT $^{* *}$ & 618 & $100 \%$ & $97 \%$ & $97 \%$ & $100 \%$ & $\mathbf{9 8 . 6 \%}$ \\
\hline IMU Sole $^{* *}$ & 618 & $100 \%$ & $89 \%$ & $97 \%$ & $100 \%$ & $\mathbf{9 6 . 4 \%}$ \\
IMU Shank $^{* *}$ & 618 & $100 \%$ & $94 \%$ & $94 \%$ & $100 \%$ & $\mathbf{9 7 . 1 \%}$ \\
IMU Sole\&Shank $^{* *}$ & 618 & $100 \%$ & $94 \%$ & $97 \%$ & $100 \%$ & $\mathbf{9 7 . 9 \%}$ \\
F/T + IMU Sole\&Shank & 1236 & $100 \%$ & $94 \%$ & $97 \%$ & $100 \%$ & $\mathbf{9 7 . 9 \%}$ \\
\hline
\end{tabular}

We decomposed the signals with the Discrete Wavelet Transformation (DWT) using the Daubechies wavelet with four vanishing moments [28].

Generally, wavelet transformations are well-suited for extracting features from a discontinuous signal as compared to Fourier transformation, for example. This is because both the frequency and the temporal information are considered. Wavelets have been previously used as features for a classification task on a bipedal robot with good results [14]. We extended this idea and also transformed the IMU signals by using DWT.

To construct the one-dimensional feature vector, we stacked the approximation coefficients of the DWTs of all sensor signals and standardized the data similar to [14]. The number of total features with respect to the selected sensor signals can be found in Table II. Alternatively, the unstacked approximation coefficients can be used as individual feature vectors. This leads to increased complexity of the training architecture due to the high amount of available sensors and was thus not followed in our work.

\section{Classification}

We trained a one-vs-one Support Vector Machine (SVM) with a linear kernel on the known soils of each of the three datasets.

SVMs are known for their robustness against overfitting, which is especially valuable since our feature vector is relatively large compared to our sample set. Size-reducing methods for the feature vector, such as Principle Component Analysis (PCA), are not needed with this classifier, which results in a highly detailed descriptor.

More complex Kernels such as quadratic or cubic SVMs yielded only marginal increases in performance and were omitted due to increased computational complexity, which would be a concern when used in a future space mission. We performed training on $80 \%$ of the data of each dataset with 5-fold cross-validation using Matlab's Statistics and Machine Learning Toolbox, keeping 20\% of the data for validation. In the next step, we tested the generalization performance by classifying the previously unknown soils. The schematic overview of our approach can be seen in Figure 5.

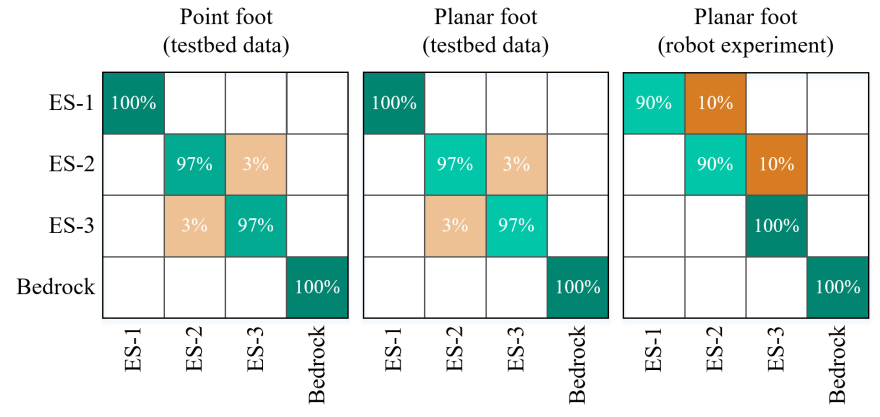

Fig. 6: Classification confusion matrices for the point and planar foot on the validation dataset of the testbed and the robot experiment.

\section{RESULTS AND DISCUSSION}

\section{A. Classification results}

The classification accuracy of more than $98 \%$ for each foot for the known soils is remarkable given the disturbances we introduced during the collection procedure on the testbed (Figure 6). The IMU signals demonstrate a marginally higher individual accuracy compared to the F/T signals even though each sensor achieves an overall accuracy well above 95\% (Table II). The classifier has no difficulties identifying the very soft ES-1 and the hard bedrock. Confusion is only present between ES-2 and ES-3, which are also similar to each other when it comes to the sinkage (Figure 7).

The classification performance of the point foot and planar foot is the same in the case that all sensor signals are used. In case only F/T signals are taken into account, the point foot achieves a slightly higher accuracy. This is intuitively clear due to the significantly higher sinkage that leads to different impact dynamics and thus more discriminative features. However, the high sinkage of the point foot design also decreases locomotion performance significantly on soft soil as shown in previous work [25].

The overall classification score achieved with the small dataset acquired by the robot is very high, with a 95\% accuracy. Misclassification occurred between ES-2 and ES-3 as well as ES-1 and ES-2. Because of the relatively small size of the validation dataset, the $10 \%$ inaccuracy results from a single misclassified event. While the predictions are generally accurate, performing multiple probing motions rather than relying on a single measurement would further increase confidence in the measurement. The experiment shows that the dedicated haptic inspection motion of the robot can be used to classify terrain in front of the robot.

\section{B. Generalization results}

So far, the classifier has only classified soils on which it was trained. On Mars, soils can be encountered in heterogeneous mixtures. Hence, we used our trained models to predict the class labels of unknown soils in order to evaluate the generalization performance. Figure 8 shows the predicted class distribution averaged over all samples. It should be noted that the point foot and planar foot results cannot be directly compared to each other since the foot soil interaction and impact dynamics are a function of the shape [7]. 


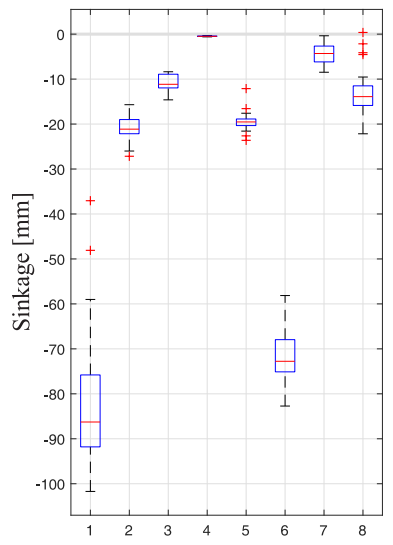

(a) Point foot sinkage

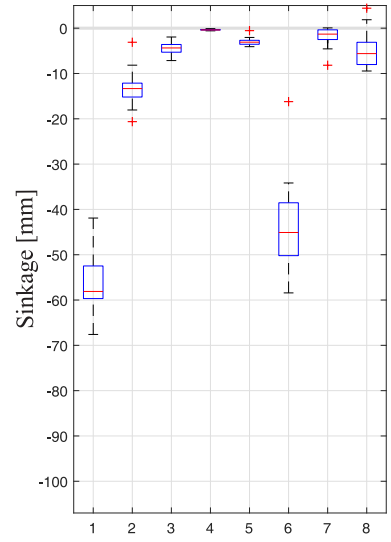

(b) Planar foot sinkage
Fig. 7: Sinkage of the point foot (a) and planar foot (b) in $\mathrm{mm}$. We performed 25 impacts on flat ES-1 (1), ES-2 (2), ES-3 (3), bedrock (4), CS (5), ES-4 variant (loose) (6), ES-4 variant (compacted) (7), and rocks placed on ES-3 (8).

The point foot can identify the hidden bedrock below ES-2 as a result of the easy penetration of the surface layer. The point foot identifies the loosely compacted ES-4 variant as ES-1 and the highly compacted ES-4 variant as bedrock, which is plausible. The CS is identified as a soil, with high probability to be ES-3, to which the soil has a strong resemblance. The point foot classifier confuses ES-1 and bedrock on the compacted ES-4 variant and ES-1 and ES-3 on the CS which have significantly different behaviors.

The planar foot creates a more plausible distribution of classification results. The classifier predicts the loose ES-4 variant as ES-1, the compacted ES-4 variant as either ES-3 or bedrock and the CS as soil with properties similar to ES-2 or ES-3. The surface layer on top of the hidden bedrock is not fully penetrated, so the classifier predicts the soil to be ES-3 or bedrock. Unclear predictions were only obtained on the rocks placed on ES-3, especially with the point foot. The rocks were exposed on the surface and thus significantly disturbed the detected signals. For instance, the foot could partially hit a rock, while partly being in contact with the soil, which results in an interesting bell-shaped probability distribution for the planar foot. We were able to accurately classify the rocks on ES-3 when added as a dedicated class, yet the generalization performance became worse and over-fitted on unknown soils.

Generally, the variety of prediction matches the qualitative assessment of the foot soil interaction.

Reproducing the results on the smaller dataset collected with the robot also follows the same trend. Variations due to slippage were not observed, since only flat soil was tested, which explains the high accuracy when detecting the bedrock.

Overall, we propose incorporating more inspection maneuvers to gain a deeper insight into the behavior of the soil and detect potential sources of error, such as rocks. The trend we observe is that our classification method generalizes well and can be used on previously unobserved soils, especially when using the planar foot design.
In the case that loose soils such as ES-1 or ES-2 would be labeled risky or inefficient for traversal, the robot would successfully manage to avoid these terrains (such as the loose ES-4 variant) with the trained classifier. On the other hand, the robot could traverse safe terrain, which visually resembles as unsafe ES-2 but has rigid bedrock underneath.

\section{CONCLUSIONS}

We show that classification of dry, granular media, in which terramechanic effects dominate, is possible. We demonstrate a haptic inspection motion with which a quadruped robot can quickly and safely analyze terrain properties by using one of its limbs. The classification approach is based on the analysis of oscillations resulting from a controlled impact on the soil. These oscillations are sensed by Force/Torque and IMU sensors in the feet. The classification is performed with a linear Support Vector Machine which uses the discret wavelet transform of the sensor signals as features. We validated the robustness of the classification approach on a dataset acquired on a dedicated single-foot testbed, which has been open sourced. Various potential disturbances such as variations in impact velocities, soil inclination, soil compaction and the occurrence of slip have been simulated.

Our method achieved an overall classification accuracy of more than $98 \%$ with a dedicated point and planar foot design. We can show that only a minimum amount of sensors is necessary in order to achieve a high classification accuracy.

The proposed inspection motion was implemented and executed on the quadruped robot ANYmal and was able to classify soils with $95 \%$ accuracy. Additionally, the performance of the prediction on previously unclassified soils shows, that a qualitative assessment of the expected performance can be carried out. For example, it was possible to distinguish hard surfaces underneath a layer of soft-looking soil or visually indistinguishable soil packing densities.

For the future, we propose to tune the impacts created on the testbed to closely match the impacts created by the robot. By training a classifier on a unified dataset acquired by testbed and robot, the method could be further robustified. Sampling on a broader variety of soils would potentially create a finer resolution on the prediction. Additionally, a measure for the confidence in the predicted class, such as the posterior probability, could be taken into account. For example, in the case of low confidence, a strategy for additional sampling, eventually with different haptic inspection motions, could be implemented. It remains to be tested whether the proprioceptive sensors of the robot could achieve the same classification accuracy.

\section{ACKNOWLEDGMENT}

The authors would like to thank Nils Funk for the mechanical design of the impact testbed, Stéphane Michaud from RUAG AG for providing the Martian soil simulants and Martin Werner Zwick from the Automation and Robotics section of the European Space Agency for providing the soil characteristics. This work has been conducted as part of ANYmal Research, a community to advance legged robotics. 


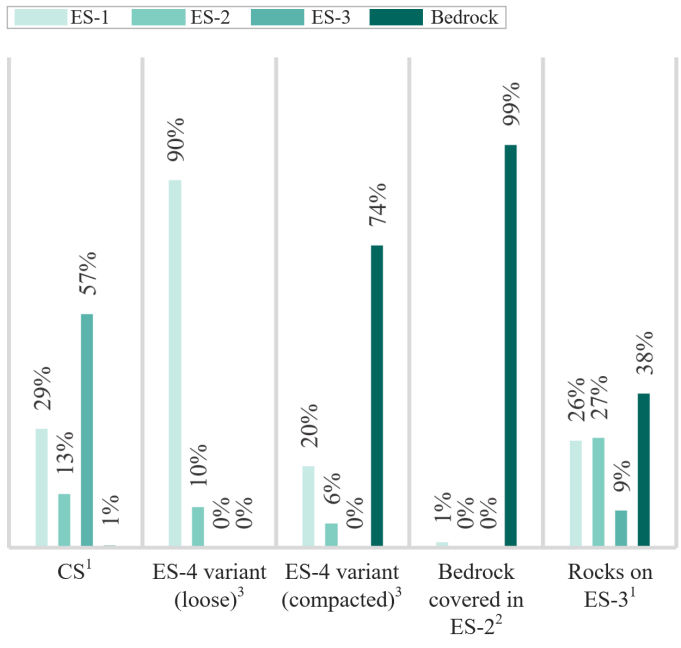

(a) Point foot (testbed data)

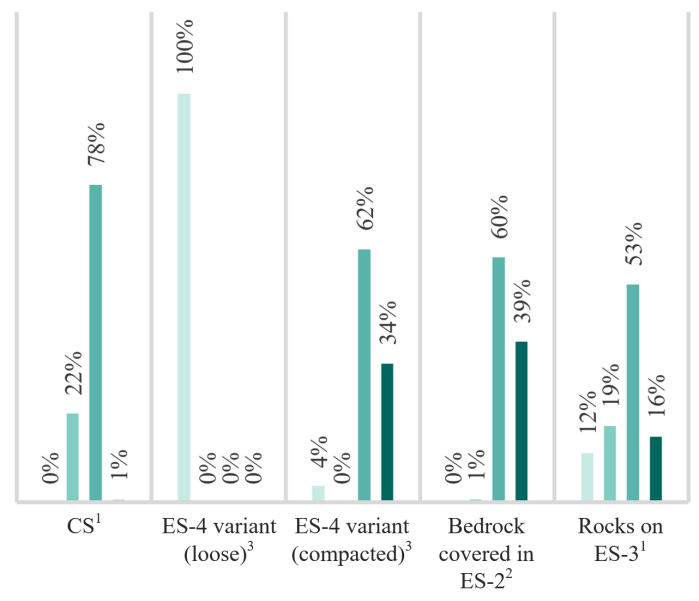

(b) Planar foot (testbed data)

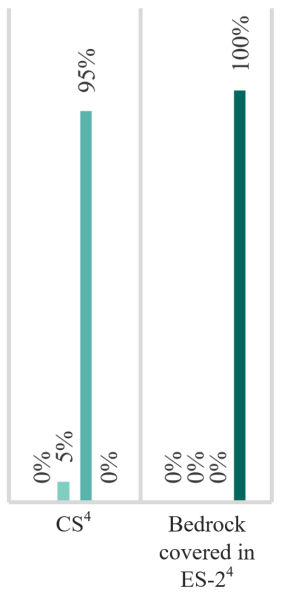

(c) Planar foot (robot experiment)

Fig. 8: The classification labels for unknown soils show that anticipated soil behavior can be predicted. It was possible to distinguish between highly compressible soils and hard surfaces. (Dataset sizes: $175^{1}, 150^{2}, 50^{3}, 20^{4}$ tests.)

\section{REFERENCES}

[1] R. E. Arvidson, et al., "Mars science laboratory curiosity rover megaripple crossings up to sol 710 in gale crater," Journal of Field Robotics, vol. 34 no. 3, pp. 495-518, 2016

[2] L. David, "Opportunity mars rover stuck in sand," Mar 2016. [Online]. Available: https://www.space.com/1019-opportunity-mars-rover-stucksand.html

[3] G. Webster and V. McGregor, "Nasa's mars rover has uncertain future as sixth anniversary nears," dec 2009. [Online]. Available: https://mars.nasa.gov/mer/newsroom/pressreleases/20091231a.html

[4] C. Cunningham, I. Nesnas, and W. Whittaker, "Improving slip prediction on mars using thermal inertia measurements," in Robotics: Science and Systems (RSS 2017), jul 2017.

[5] C. Brunskill, et al., "Characterisation of martian soil simulants for the exomars rover testbed," Journal of Terramechanics, vol. 46, pp. 419-438, dec 2011.

[6] J.-Y. Wong and A. Reece, "Prediction of rigid wheel performance based on the analysis of soil-wheel stresses part i. performance of driven rigid wheels," Journal of Terramechanics, vol. 4, no. 1, pp. 81 - 98, 1967.

[7] L. Ding, et al., "Foot-terrain interaction mechanics for legged robots: Modeling and experimental validation," The International Journal of Robotics Research, vol. 32, no. 13, pp. 1585-1606, 2013.

[8] C. A. Brooks and K. Iagnemma, "Vibration-based terrain classification for planetary exploration rovers," IEEE Transactions on Robotics, vol. 21, no. 6, pp. 1185-1191, dec 2005.

[9] I. Halatci, C. A. Brooks, and K. Iagnemma, "Terrain classification and classifier fusion for planetary exploration rovers," in 2007 IEEE Aerospace Conference, mar 2007, pp. 1-11.

[10] C. A. Brooks and K. Iagnemma, "Self-supervised terrain classification for planetary surface exploration rovers," Journal of Field Robotics, vol. 29, no. 3, pp. 445-468, 2012.

[11] F. Comin and C. Saaj, "Planetary soil classification based on the analysis of the interaction with deformable terrain of a wheel-legged robot," in IEEE/RSJ International Conference on Intelligent Robots and Systems (IROS 2015), sep 2015, pp. 5460-5465.

[12] K. Walas, "Terrain classification and negotiation with a walking robot," Journal of Intelligent \& Robotic Systems, vol. 78, no. 3, pp. 401-423, jun 2015.

[13] J. Mrva and J. Faigl, "Feature extraction for terrain classification with crawling robots," Information Technologies - Applications and Theory (ITAT 2015), vol. 1422, pp. 179-185, sep 2015.

[14] K. Walas, D. Kanoulas, and P. Kryczka, "Terrain classification and locomotion parameters adaptation for humanoid robots using force/torque sensing," in IEEE-RAS 16th International Conference on Humanoid Robots (Humanoids 2016), nov 2016, pp. 133-140.

[15] M. Hoffmann, K. Štěpánová, and M. Reinstein, "The effect of motor action and different sensory modalities on terrain classification in a quadruped robot running with multiple gaits," Robotics and Autonomous Systems, vol. 62, no. 12, pp. 1790 - 1798, 2014.

[16] M. A. Hoepflinger, C. D. Remy, M. Hutter, L. Spinello, and R. Siegwart, "Haptic terrain classification for legged robots," in IEEE/RSJ International Conference on Robotics and Automation (ICRA 2010), may 2010, pp. 2828-2833.

[17] K. Walas, "Tactile sensing for ground classification," Journal of Automation, Mobile Robotics \& Intelligent Systems, vol. 7, pp. 18 -, jan 2013.

[18] P. Dallaire, K. Walas, P. Giguère, and B. Chaib-draa, "Learning terrain types with the pitman-yor process mixtures of gaussians for a legged robot," in IEEE/RSJ International Conference on Intelligent Robots and Systems (IROS 2015), sep 2015, pp. 3457-3463.

[19] J. Degrave, R. Van Cauwenbergh, F. Wyffels, T. Waegeman, and B. Schrauwen, "Terrain classification for a quadruped robot," in 12th International Conference on Machine Learning and Applications (ICMLA 2013). IEEE, 2013, pp. 185-190.

[20] C. Kertész, "Rigidity-based surface recognition for a domestic legged robot," IEEE Robotics and Automation Letters, vol. 1, no. 1, pp. 309-315, jan 2016.

[21] L. Wellhausen, A. Dosovitskiy, R. Ranftl, K. Walas, C. Cadena, and M. Hutter, "Where should i walk? predicting terrain properties from images via self-supervised learning," IEEE Robotics and Automation Letters, vol. 4, no. 2, 2019.

[22] H. Kolvenbach, M. Breitenstein, C. Gehring, and M. Hutter, "Scalability analysis of legged robots for space exploration," in 68th International Astronautical Congress (IAC). IAF, 2017.

[23] P. Arm, et al., "Spacebok: A dynamic legged robot for space exploration," in IEEE/RSJ International Conference on Robotics and Automation (ICRA 2019), May 2019.

[24] M. Hutter, et al., "Anymal - toward legged robots for harsh environments," Advanced Robotics, vol. 31, no. 17, pp. 918-931, 2017.

[25] H. Kolvenbach, D. Bellicoso, F. Jenelten, L. Wellhausen, and M. Hutter, "Efficient gait selection for quadrupedal robots on the moon and mars," 14th International Symposium on Artificial Intelligence, Robotics and Automation in Space (i-SAIRAS 2018), June 2018.

[26] R. Käslin, H. Kolvenbach, L. Paez, K. Lika, and M. Hutter, "Towards a passive adaptive planar foot with ground orientation and contact force sensing for legged robots," IEEE/RSJ International Conference on Intelligent Robots and Systems (IROS 2018), Oct. 2018.

[27] P. Fankhauser, C. D. Bellicoso, C. Gehring, R. Dubé, A. Gawel, and M. Hutter, "Free gait an architecture for the versatile control of legged robots," in IEEE-RAS 16th International Conference on Humanoid Robots (Humanoids 2016), nov 2016, pp. 1052-1058.

[28] I. Daubechies, Ten Lectures on Wavelets. Society for Industrial and Applied Mathematics, 1992. 